\title{
ЭКСПРЕССИЯ НЕЙРОЭНДОКРИННЫХ МАРКЕРОВ ПРИ ЭНДОМЕТРИОИДНОМ И СЕРОЗНОМ РАКЕ ЯИЧНИКОВ
}

\section{EXPRESSION OF NEUROENDOCRINE MARKERS IN ENDOMETRIOID AND SEROUS OVARIAN CANCER}

\section{K. Yagafarova \\ G. Raskin \\ V. Vinokurov \\ M. Mukhina}

Summary. This work analyzes the expression of neuroendocrine markers in endometrioid and serous ovarian cancer. The median Ki67 index was significantly higher in the group with endometrioid adenocarcinoma of ovarian cancer, amounting to $55(20 ; 80)$, against the median value of $30(10 ; 52.5)$ in the group of serous ovarian adenocarcinoma ( $p<0.05$ ). A pronounced variability of ChrA was revealed, the maximum concentrations were recorded in patients with endometriodal adenocarcinoma ( $p<0.01)$. A positive value of INSM1 was found in 7 patients (9.59\%), of which 5 patients with endometriotic ovarian adenocarcinoma (17.24\% in the group) and 2 patients with serous adenocarcinoma ( $4.55 \%$ in the group). It was revealed that the expression of neuroendocrine markers: synaptophysin, chromogranin A, CD56 in ovarian adenocarcinomas is not a manifestation of the neuroendocrine nature of the tumor, since it is not associated with the expression of INSM1, but the expression of synaptophysin is a poor prognostic sign and is associated with worse survival.

Keywords: chromogranin A (ChrA), synaptophysin, CD56, type 2 somatostatin, type 5 somatostatin, INSM1, ovarian cancer.
Ягафарова Карина Робертовна

Врач-онколог, аспирант, Российский научный чентр радиологии и хирургических технологий имени академика А.М. Гранова vahitova-20@mail.ru

Раскин Григорий Александрович

Д.м.н., дочент, Российский научный чентр радиологии и хирургических технологий имени академика А.М. Гранова

Винокуров Владимир Леонидович Д.м.н., профессор, Российский научный чентр радиологии и хирургических технологий имени академика А.М. Гранова

Мухина Марна Семеновна

К.м.н., С.н.С., Российский научный чентр радиологии и хирургических технологий имени академика

А.М. Гранова

Аннотация. В данной работе проведен анализ экспрессии нейроэндокринных маркеров при эндометриоидном и серозном раке яичников. Медиана индекса Ki67 была значительно выше в группе с эндометриоидной аденокарциномой рака яичников, составив 55 (20;80), против значения медианы $30(10 ; 52,5)$ в группе серозной аденокарциномы яичников $(p<0,05)$. Выявлена выраженная вариабельность ХгА, максимальные концентрации зафиксированы у больных с эндометриодной аденокарциномой ( $p<0,01)$. Положительное значение INSM1 выявлено у 7 пациенток (9,59\%), из которых 5 больных с эндометриодной аденокарциномой яичников (17,24\% в группе) и 2 пациентки с серозной аденокарциномой (4,55\% в группе). Выявлено, что экспрессия нейроэндокринных маркеров: синаптофизин, хромогранин A, CD56 в аденокарциномах яичников не является проявлением нейроэндокринной природы опухоли, так как не связано с экспрессией INSM1, но экспрессия синаптофизина является плохим прогностическим признаком и связана с худшей выживаемостью.

Ключевые слова: хромогранин $A(X г A)$, синаптофизин, $C D 56$, соматостатин 2 типа, соматостатин 5 типа, INSM1, рак яичников.

молекулярно-биологических маркеров, которые помогут определить биологические особенности опухоли, прогнозировать развитие метастазов, реакции опухоли на лечение и позволят составить индивидуальный прогноз [4].

\section{Lе^ь исслеАования}

Анализ экспрессии нейроэндокринных маркеров при эндометриоидном и серозном раке яичников. 
Таблица 1. Оценка частоты встречаемости нейроэндокринных маркеров при аденокарциномах яичников различной гистологической структуры

\begin{tabular}{|l|l|l|l|l|}
\hline Маркеры & CAя $(\mathbf{n = 4 4 )}$ & ME-1 & ЭAЯ (n=29) & ME-2 \\
\hline Индекс Кі 67 & $34,89 \pm 27,88$ & $30(10 ; 52,5)$ & $53,00 \pm 31,35$ & $55(20 ; 80)$ \\
\hline Хромогранин A & $1,52 \pm 3,30$ & $0(0 ; 0,5)$ & $10,76 \pm 20,10$ & $0(0 ; 10)$ \\
\hline Синаптофизин & $10,64 \pm 23,54$ & $0(0 ; 3)$ & $9,93 \pm 20,07$ & $0(0 ; 3)$ \\
\hline CD56 & $38,18 \pm 36,79$ & $37,5(0 ; 75)$ & $31,76 \pm 36,35$ & $5(1 ; 60)$ \\
\hline Соматостатин 2 типа & $1,59 \pm 4,70$ & $0(0 ; 0,5)$ & $8,17 \pm 18,83$ & $0(0 ; 2)$ \\
\hline Соматостатин 5 типа & $5,64 \pm 14,81$ & $0(0 ; 0)$ & $12,41 \pm 25,27$ & $0(0 ; 10)$ \\
\hline INSM1 & $2,05 \pm 12,12$ & $0(0 ; 0)$ & $5,00 \pm 17,22$ & $0(0 ; 0)$ \\
\hline
\end{tabular}

Box \& Whisker Plot: KI_67

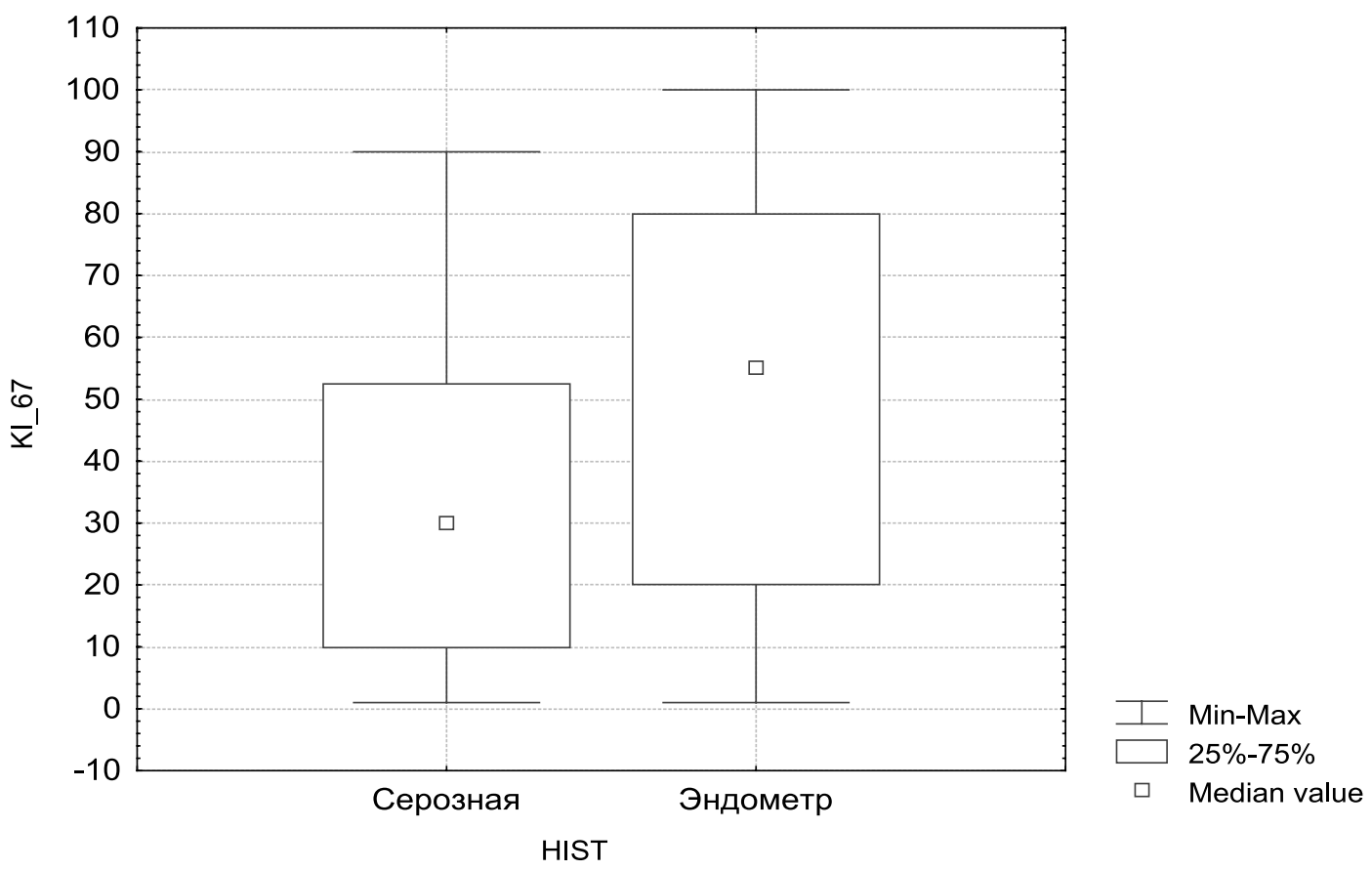

Рис. 1. Взаимосвязь между гистологическим типом опухоли и экспрессией индекса Кі67\%

\section{Материалы и метолы}

В период с 2009 по 2018 г. было проведено сравнительное ретроспективное исследование на базе ФГБУ «Российский научный центр радиологии и хирургических технологий имени академика А.М.Гранова» и СПбГБУЗ «Городской клинический онкологический диспансер» Материалом для исследования послужили результаты наблюдений за 73 пациентками с морфологически подтвержденным диагнозом: рак яичников.

Иммуногистохимическое исследование осуществляли на парафиновых срезах толщиной 3-4 мкм авидин-биотин-пероксидазным методом по стандартной методике с использованием первичных антител [1]
Статистический анализ проводили в программе Statistica 10 (Statsoft, США). Для сравнения количественных характеристик в группах применялся дисперсионный анализ, достоверность различий определялась с использованием непараметрического критерия Манна-Уитни. Статистически значимыми считались различия при $\mathrm{p}<0,05$.

\section{Результаты и обсужление}

Все больные были разделены в зависимости от варианта гистологического строения опухоли на две группы. В первую группу вошли 29 пациенток с эндометриоид-

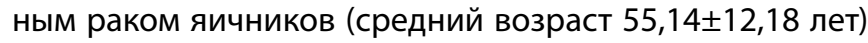
у $89,66 \%$ которых выявлена II стадия заболевания 
Histogram: KI_67
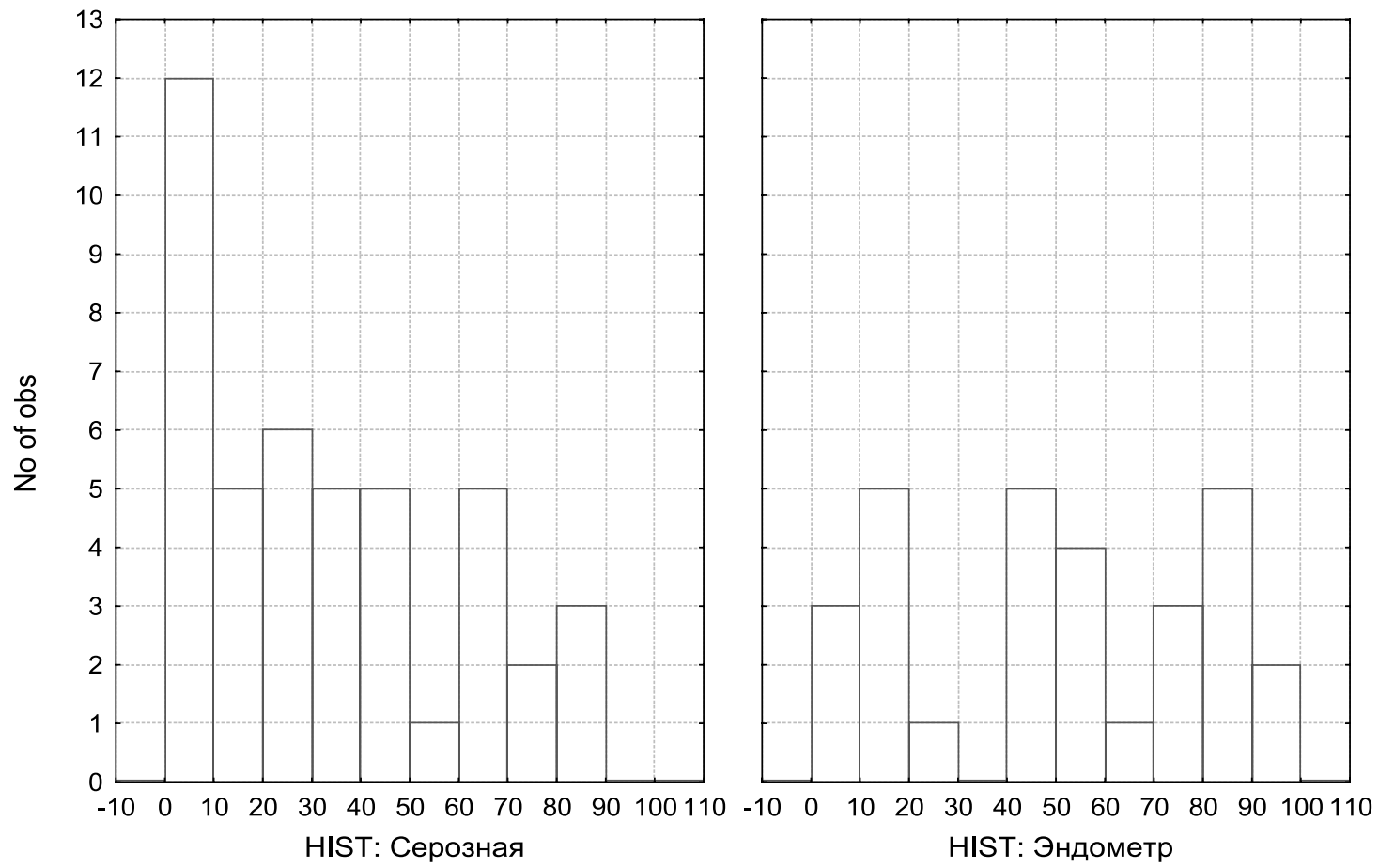

Рис. 2. Особенности экспрессии Кі 67 при различных гистотипах опухоли

и у 10,34\% - I стадия. Вторую группу составили 44 пациентки с серозным раком яичников высокой степени

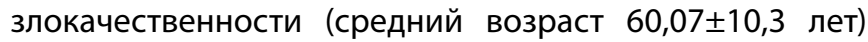
у 70,45\% которых выявлена III стадия заболевания и у 29,55\% - IV стадия. У 60,27\% больных наблюдались опухоли высокой степени злокачественности, у 31,51\% - опухоли с умеренной степенью дифференцировки и в 8,22\% случаев выявлены высокодифференцированные опухоли с низкой степенью злокачественности.

Изучались различные маркеры нейроэндокринной дифференцировки, такие, как хромогранин А (ХгА), синаптофизин, CD56, соматостатин 2 типа, соматостатин 5 типа и INSM1 (табл. 1).

Примечание: САЯ - Серозная аденокарцинома яичников; ME-1 интерквартильная широта (LQ; UQ) для серозных аденокарцином; ЭАЯ - Эндометриоидная аденокарцинома яичников; ME-2 интерквартильная широта (LQ; UQ) для эндометриоидных аденокарцином

Особое значение в прогнозировании поведения опухоли придается ее пролиферативной активности. Наиболее распространенный маркер пролиферации антиген Кі-67, предположительно относящийся к регуляторным белкам [6].
Кі-67-позитивные клетки выявляли во всех случаях, медиана индекса Кі 67 составила $30(10 ; 52,5)$ в группе серозной аденокарциномы яичников и $55(20 ; 80)$ в группе с эндометриоидным раком яичников, $\mathrm{p}<0,05$ (рис. 1,2).

Хромогранин А - гликопротеин, который показывает высокую корреляцию с ультраструктурными признаками нейроэндокринной дифференцировки, причем в карциноидах отмечается большее количество хромогранин А-позитивных гранул, чем в мелкоклеточном раке [7].

В результате исследования выявлена выраженная вариабельность ХгА, при этом максимальные концентрации были зафиксированы у больных с эндометриодной аденокарциномой, $p<0,01$ (рис. 3,4).

Учитывая полученные данные, можно сделать вывод, что у больных с эндометриодной аденокарциномой яичников может выявляться высокая экспрессия хромогранина $(p<0,01)$.

Также проводилось сравнение экспрессии иммуногистохимических маркеров у больных аденокарциномами яичников в зависимости от показателя INSM1 (Insulinoma-Associated Protein1) - диагностического маркера нейроэндокринных опухолей (табл. 2). 


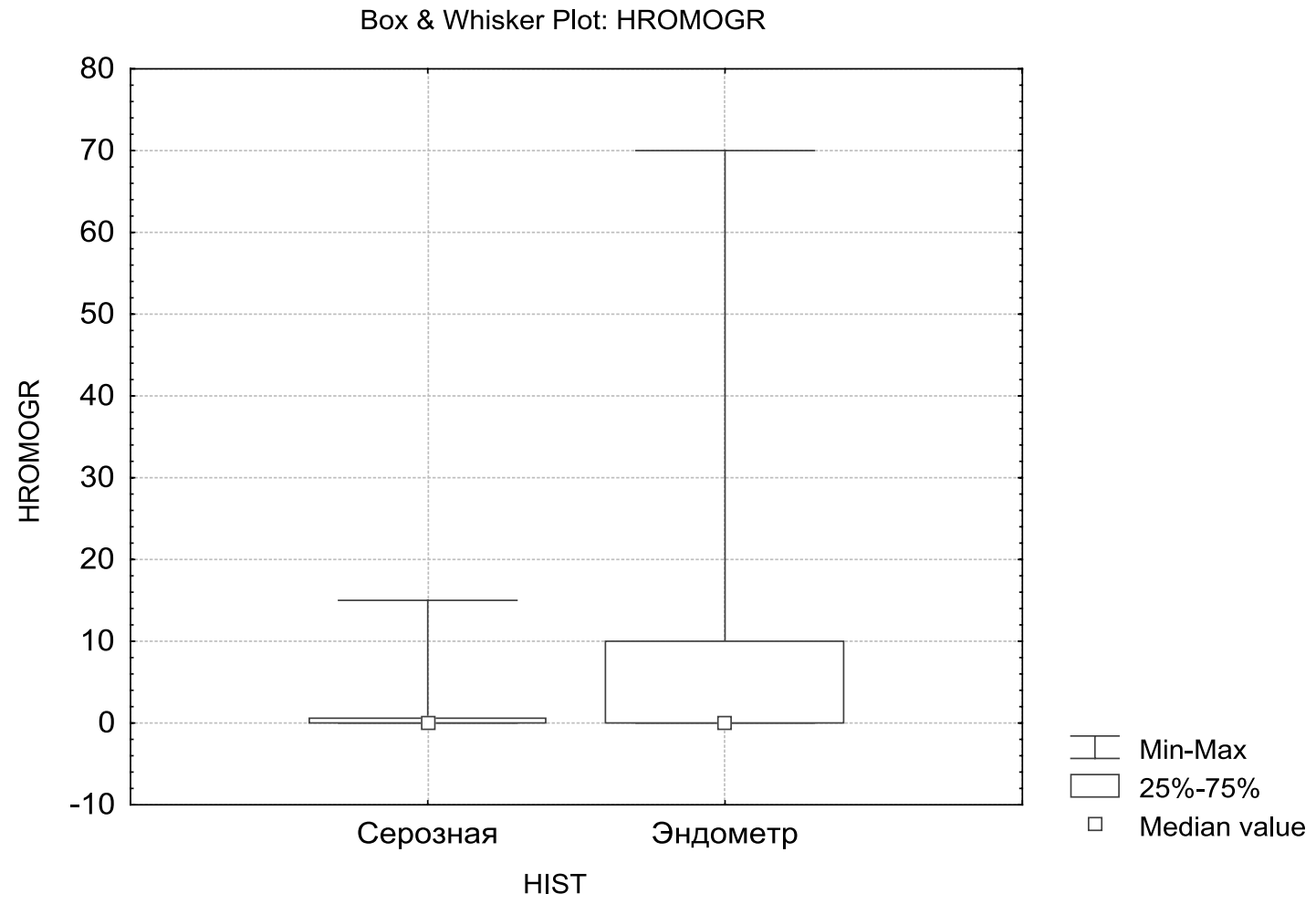

Рис. 3. Взаимосвязь между гистологическим типом опухоли и экспрессией хромогранина

Histogram: HROMOGR
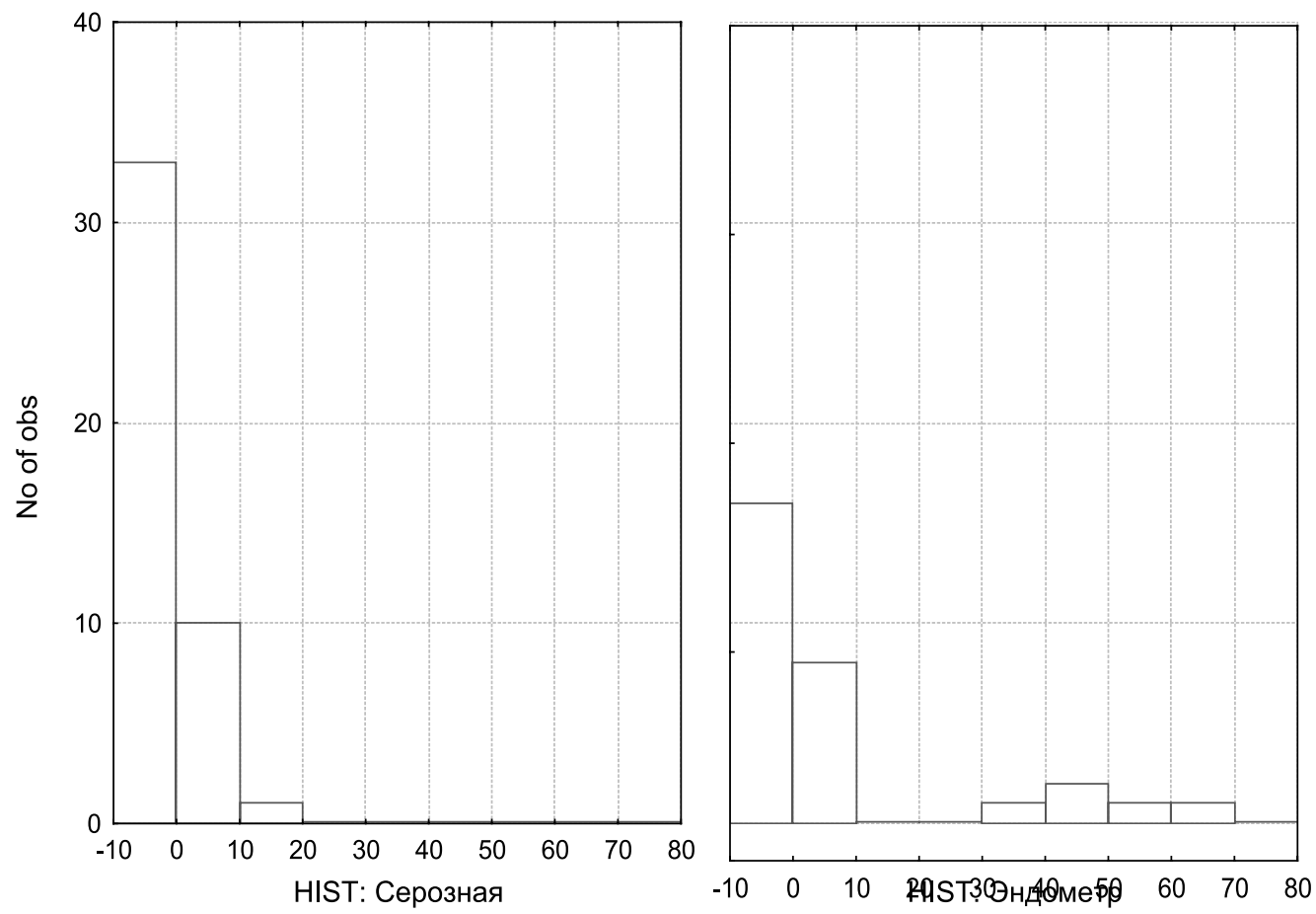

Рис. 4. Особенности экспрессии хромогранина при различных гистотипах опухоли 
Таблица 2. Сравнение экспрессии иммуногистохимических маркеров у больных аденокарциномами яичников в зависимости от показателя INSM1

\begin{tabular}{|c|c|c|c|c|c|}
\hline Маркеры & $\begin{array}{l}\text { INSM1 }=0 \\
(n=66)\end{array}$ & $\begin{array}{l}\text { INSM1=0 } \\
\text { ME (LQ; UQ) }\end{array}$ & $\begin{array}{l}\text { INSM1 } 1>0 \\
(n=7)\end{array}$ & $\begin{array}{l}\text { INSM1>0 } \\
\text { ME (LQ; UQ) }\end{array}$ & $p$ \\
\hline Индекс Кі 67 & $41,21 \pm 30,44$ & $38(15 ; 70)$ & $50,29 \pm 31,45$ & $50(20 ; 80)$ & $p=0,4$ \\
\hline Хромогранин А & $3,92 \pm 10,25$ & $0(0 ; 5)$ & $17,14 \pm 29,84$ & $0(0 ; 50)$ & $p=0,1$ \\
\hline Синаптофизин & $9,48 \pm 21,40$ & $0(0 ; 3)$ & $18,57 \pm 28,39$ & $0(0 ; 35)$ & $p=0,5$ \\
\hline CD56 & $33,56 \pm 35,70$ & $15(0 ; 60)$ & $55,14 \pm 41,02$ & $70(4 ; 92)$ & $p=0,2$ \\
\hline $\begin{array}{l}\text { Соматостатин } \\
2 \text { типа }\end{array}$ & $3,53 \pm 10,52$ & $0(0 ; 1)$ & $10,57 \pm 26,22$ & $0(0 ; 2)$ & $p=0,2$ \\
\hline $\begin{array}{l}\text { Соматостатин } \\
5 \text { типа }\end{array}$ & $9,14 \pm 20,62$ & $0(0 ; 3)$ & $0,71 \pm 1,89$ & $0(0 ; 0)$ & $p=0,3$ \\
\hline
\end{tabular}

Таблица 3. Корреляционный анализ экспрессии нейроэндокринных маркеров

\begin{tabular}{|l|l|l|l|}
\hline Корреляционная связь & Bсего $(\mathbf{n})$ & R-коэффициент корреляции & p-level \\
\hline \multirow{2}{*}{$\begin{array}{l}\text { Синаптофизин и соматостатин } \\
\text { типа }\end{array}$} & Bсе пациентки $(\mathrm{n}=73)$ & 0,237 & 0,043 \\
\cline { 2 - 4 } & INSM1 $=0(\mathrm{n}=66)$ & 0,254 & 0,040 \\
\hline Синаптофизин и хромогранин A & INSM1 >1 (n=7) & 0,836 & 0,019 \\
\hline
\end{tabular}

В результате исследования выявлено 7 пациенток с положительным значением INSM1 (9,59\%), из которых 5 больных с эндометриодной аденокарциномой яичников (17,24\% в группе) и 2 пациентки с серозной аденокарциномой (4,55\% в группе). Достоверно значимых корреляций INSM1 с другими иммуногистохимическими маркерами не выявлено ( $p>0,05)$.

Корреляционный анализ экспрессии нейроэндокринных маркеров для всех пациенток с аденокарциномой яичников позволил выявить значимую корреляционную связь только между показателями синаптофизина и соматостатина 2 типа ( $\mathrm{R}=0,237 ; \mathrm{p}=0,043)$, эти же показатели коррелировали в группе пациенток с отрицательным INSM1 ( $R=0,254 ; p=0,040)$ (табл. 3).

В группе INSM1 >1 выявлена значимая корреляционная связь между показателями экспрессии синаптофизина и хромогранина $A(R=0,836 ; p=0,019)$.

\section{Выво $\triangle \mathrm{b}$}

Результаты анализа пролиферативной активности показали, что медиана индекса Ki67 была значительно выше в группе с эндометриоидной аденокарциномой яичников, составив 55 (20;80), против значения медианы $30(10 ; 52,5)$ в группе серозной аденокарциномы яичников $(p<0,05)$.

В результате исследования выявлена выраженная вариабельность ХгА, при этом максимальные концентра- ции были зафиксированы у больных с эндометриодной аденокарциномой $(\mathrm{p}<0,01)$.

Положительное значение INSM1 выявлено у 7 пациенток (9,59\%), из которых 5 больных с эндометриодной аденокарциномой яичника (17,24\% в группе) и 2 пациентки с серозной аденокарциномой (4,55\% в группе).

В результате исследования не выявлена связь экспрессии INSM1 с экспрессией нейроэндокринных маркеров: синаптофизина, хромогранина A, CD56, соматостатина 2 типа, соматостатина 5 типа и индекса Ki 67 в аденокарциномах яичников ( $p>0,05)$.

Корреляционный анализ экспрессии нейроэндокринных маркеров позволил выявить значимую корреляционную связь между показателями синаптофизина и соматостатина 2 типа ( $R=0,237 ; p=0,043)$, эти же показатели коррелировали в группе пациенток отрицательным INSM1 ( $R=0,254 ; p=0,040)$. В группе INSM1 >1 выявлена значимая корреляционная связь между показателями экспрессии синаптофизина и хромогранина $A(R=0,836$; $\mathrm{p}=0,019)$.

Таким образом, экспрессия нейроэндокринных маркеров: синаптофизин, хромогранин A, CD56 в аденокарциномах яичников не является проявлением нейроэндокринной природы опухоли, так как не связана с экспрессией INSM1, но экспрессия синаптофизина является плохим прогностическим признаком и связана с худшей выживаемостью. 


\section{ЛИТЕРАТУРА}

1. Князева М.В., Прокопюк А. В. Новые подходы к проблеме повышения эффективности лечения больных раком яичников поздних стадий. Научный взгляд в будущее. 2019; 1(13):119-124.

2. Цандекова М. Р. Современные представления о факторах прогноза при раке яичника. Успехи современной науки и образования. 2016; 6(11):99-106.

3. Kurman R.J., Shih I. M. The Dualistic Model of Ovarian Carcinogenesis. Am. J. Pathol. 2016;186:733-747.

4. Маршутина Н.В., Солохина М. П., Алентов И. И., Сергеева Н. С. Клиническая значимость биологических маркеров при раке яичников, раке предстательной железы, колоректальном раке. Исследования и практика в медицине. 2016;3(1):46-57.

5. Dabbs D. J. Diagnostic Immunohistochemistry. Elsevier Science, 2010.

6. Шацева Т.А., Мухина М. С. Антиген Кі-67 в оценке опухолевой пролиферации. Его структура и функции. Вопросы онкологии. 2004; 50(2): 157-64.

7. Dardick l., Christensen H., Stratis M. Immunoelectron microscopy for chromogranin A in small cell neuroendocrine carcinoma of lung. Utrastruct. pathol. 1996;20:361-368.

( Я Ягафарова Карина Робертовна ( vahitova-20@mail.ru ), Раскин Григорий Александрович,

Винокуров Владимир Леонидович, Мухина Марна Семеновна.

Журнал «Современная наука: актуальные проблемы теории и практики»

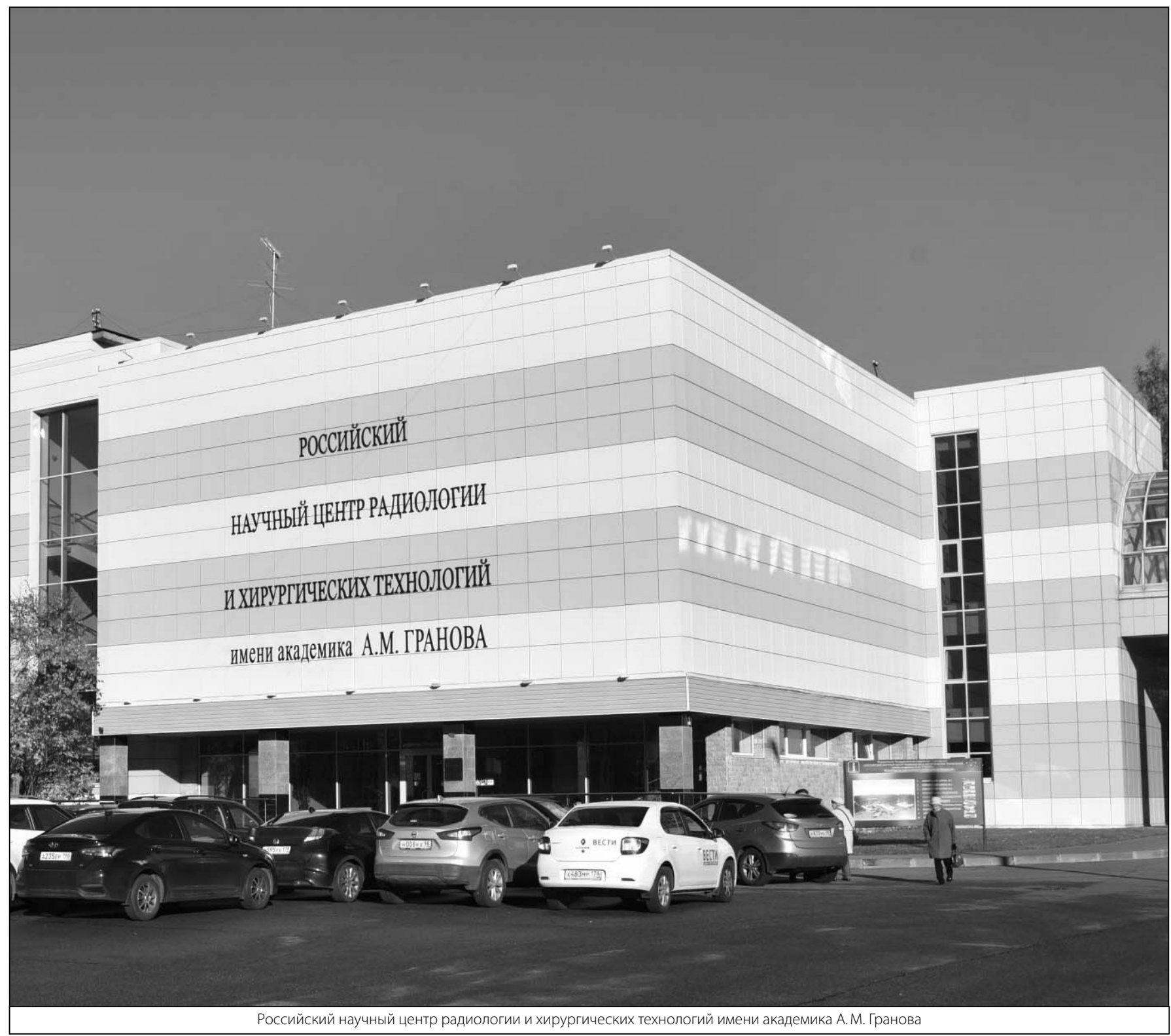

